\title{
Performance of Wideband CDMA Systems Supporting Multimedia Traffic
}

\author{
Michela Meo, Associate Member, IEEE, and Emanuele Viterbo, Member, IEEE,
}

\begin{abstract}
In this letter we evaluate the performance of wideband CDMA cellular systems providing different classes of multimedia traffic and supporting user mobility. A Markovian teletraffic model of the user dynamics is developed. Constraints are imposed in the model which account for the multiple access interference among active users. Results are shown in terms of call blocking probabilities and average number of active connections. The methodology proposed is a useful tool for the design and planning of third generation cellular systems.
\end{abstract}

Index Terms-CDMA mobile systems, Markovian models, multimedia traffic.

\section{NOMENCLATURE}

A cell of the system is defined by the following parameters.

$C \quad$ Number of distinct classes of service provided to users (correspondingly users are partitioned into $C$ classes).

$L_{i} \quad$ Spreading factor for class $i$.

$L_{\max } \quad$ Maximum spreading factor corresponding to the basic bit rate $R_{b}$.

$E_{b} \quad$ Energy per transmitted bit (a perfectly power controlled system is assumed).

$N_{0} \quad$ Noise power spectral density plus interference from neighboring cells.

$\lambda_{i} \quad$ Generation rate of new class $i$ calls from users residing in the cell.

$\lambda_{i, h} \quad$ Arrival rate of incoming handover requests from neighboring cells for class $i$ calls.

$1 / \mu_{i} \quad$ Average duration of class $i$ calls.

$1 / \mu_{h} \quad$ Average time a user spends in a cell (dwell time); this parameter derives from the user mobility and it is independent from the class of service which a user is possibly employing.

\section{INTRODUCTION}

$\mathbf{I}$ $\mathrm{N}$ THIS letter we study the performance of a wideband CDMA cellular system providing different classes of traffic such as voice, video and data. Performance is assessed in terms of blocking probability and average number of users per cell taking into account user mobility and traffic characteristics. The proposed dynamic model of the users in the system is based on Markovian assumptions and focuses on the behavior of a single cell. It approximates the interaction between adjacent cells by

Manuscript received December 15, 2000. The associate editor coordinating the review of this letter and approving it for publication was Dr. S. Batalama. This work was presented in part at ISITA 2000, Honolulu, HI, November 2000.

The authors are with the Dipartimento di Elettronica, Politecnico di Torino, Turin 10129, Italy (e-mail: michela@polito.it; viterbo@polito.it).

Publisher Item Identifier S 1089-7798(01)05509-0. means of the incoming handover flow, which is assumed to be equal to the handover flow out of the considered cell and it is derived by a fixed point iterative procedure.

We consider the uplink of a single cell of a UMTS-like system where user terminals access the base station with different bit rates according to the requested service. The system is essentially limited by the multiple access interference (MAI) among active users. The different classes of users are assigned different spreading factors according to their bit-rate, in turn, this entails different amounts of MAI.

Similar models have been proposed in the literature for the design and planning of mobile cellular telephony networks (see for example [1]-[5]). In [6], [7] this approach was employed taking into account the multiple access interference in CDMA systems. With respect to these last two papers, the novelty of our results is twofold. We extend the approach to different classes of users employing different spreading factors and we consider the presence of user mobility.

\section{SYSTEM MODEL}

The dynamics that govern the cell behavior are the arrival processes of call requests (either new calls or incoming handovers) and of channel releases (either because of a call termination or because of an outgoing handover).

In the development of the model, the arrival processes are assumed to be Poisson; let $\lambda_{i}$ and $\lambda_{i, h}$ be the parameters of the Poisson processes which model respectively new call arrivals and incoming handover requests for class $i$ calls. The call duration and the dwell time are random variables with negative exponential distribution with parameter $\mu_{i}$ and $\mu_{h}$, respectively.

The class $i$ arrival process is the superposition of the arrival process of new call requests and of incoming handovers; the resulting total arrival rate is $\lambda_{i}+\lambda_{i, h}$. The channel holding time is the interval between the instant in which a user starts using the channel and the instant in which the channel is released either because of the call termination or because the user is moving out of the cell. The channel holding time is defined as the minimum between the call residual time and the dwell time. Due to the assumptions introduced above, the call residual time has the same distribution as the call duration (negative exponential with parameter $\mu_{i}$ for class $i$ calls). The channel holding time is thus an exponentially distributed random variable with parameter $\mu_{i}+\mu_{h}$.

Given the assumptions above, we develop a Markovian model of a cell whose state is defined by the vector 
$\mathbf{u}=\left(u_{1}, u_{2}, \ldots, u_{C}\right)$ where each component $u_{i}$ represents the number of active class $i$ connections.

Following the approach of [6] we assume that each user in class $i$ has an error probability which is a decreasing function (depending on the channel model) of the signal-to-interference ratio (SIR)

$$
\operatorname{SIR}_{i}=\frac{E_{b}}{N_{0}+\sum_{j=1, j \neq i}^{C} u_{j} E_{b} / L_{j}+\left(u_{i}-1\right) E_{b} / L_{i}} .
$$

Each class has an outage error probability $P_{i}^{*}$ above which the corresponding service cannot be reliably supplied. This corresponds to the limiting value $\operatorname{SIR}_{i}^{*}$. The system should not accept a call whenever its admission would produce an outage to the currently active users due to the higher interference level. The probability that a call is refused is called blocking probability and is one of the main performance measures of the system. Hence the number of users per class must satisfy the following relations:

$$
\mathrm{SIR}_{i} \geq \mathrm{SIR}_{i}^{*}, \quad i=1, \ldots, C .
$$

The model state space is obtained by substituting (1) into (2):

$$
\mathcal{S}=\left\{\mathbf{u}=\left(u_{1}, u_{2}, \ldots, u_{C}\right) \mid \sum_{j=1}^{C} \frac{u_{j}}{L_{j}} \leq K\right\}
$$

where

$$
K=\min _{i}\left(\frac{1}{\operatorname{SIR}_{i}^{*}}+\frac{1}{L_{i}}-\frac{N_{0}}{E_{b}}\right) .
$$

As an example, the state space for the scenario considered in next section, where voice and data services are provided, is shown in Fig. 1. Circles stand for states and the straight line represents the limiting condition.

Since this system admits a product-form solution, the steadystate probabilities $\pi(\mathbf{u}) \mathrm{s}$ are computed as the product of the single-class solutions:

$$
\pi(\mathbf{u})=\pi\left(\mathbf{u}_{0}\right) \prod_{i=1}^{C} \frac{\rho_{i}^{u_{i}}}{u_{i} !}
$$

with

$$
\pi\left(\mathbf{u}_{0}\right)=\pi(0,0, \ldots, 0)=\left(\sum_{\mathbf{u} \in \mathcal{S}} \prod_{i=1}^{C} \frac{\rho_{i}^{u_{i}}}{u_{i} !}\right)^{-1}
$$

where $\rho_{i}$ is the class $i$ traffic: $\rho_{i}=\left(\lambda_{i}+\lambda_{h, i}\right) /\left(\mu_{i}+\mu_{h}\right)$.

The incoming handover flows $\lambda_{h, i} \mathrm{~s}$ are not a priori known and have to be derived from the model by means of a fixed-point procedure. This procedure is based on the assumption that under steady-state conditions the flow of outgoing handover requests, $\lambda_{h, i}^{\text {(out) }}$, equals the flow of incoming handover requests, $\lambda_{h, i}$.

The flow of outgoing handover requests for class $i$ calls is obtained from the model

$$
\lambda_{h, i}^{(\text {out })}=E\left[u_{i}\right] \mu_{h}
$$

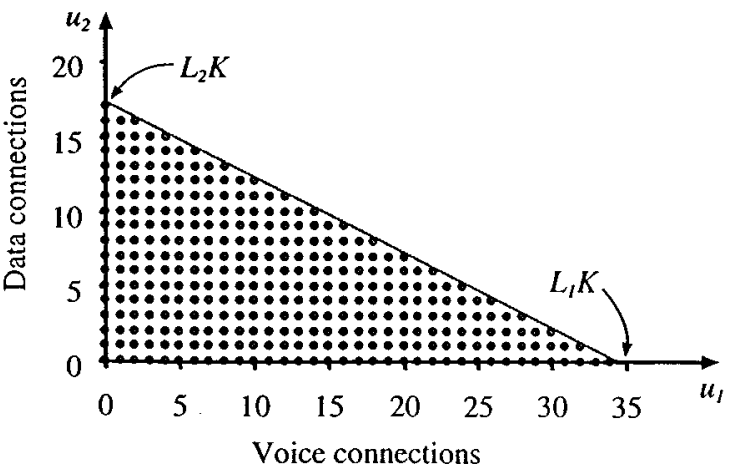

Fig. 1. State space for the voice and data scenario.

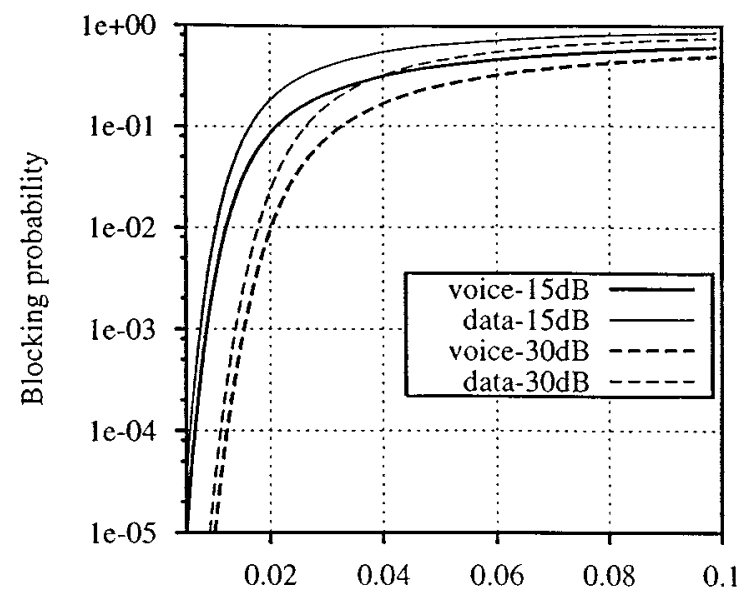

Traffic $\lambda_{2}=\lambda_{1} / 5$

Fig. 2. Voice and data blocking probability versus data call arrival rate for $E_{b} / N_{0}=15,30$

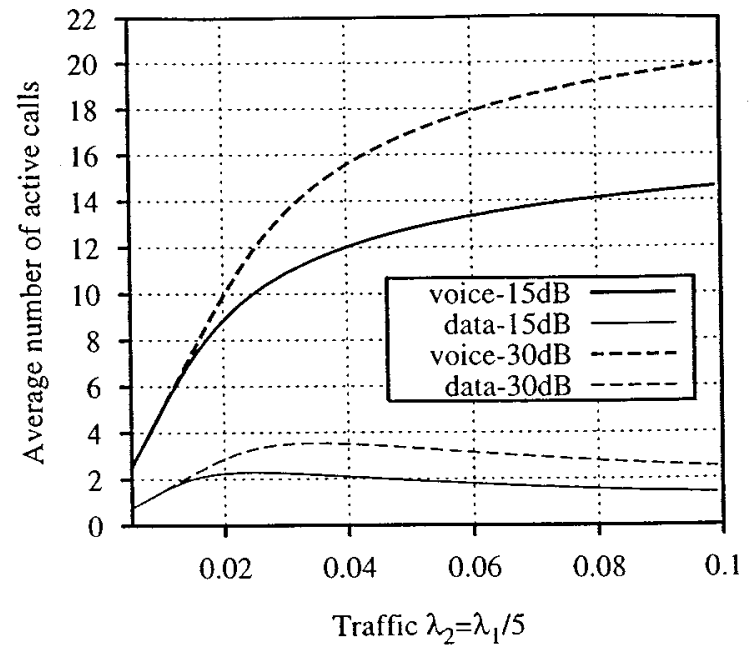

Fig. 3. Average number of active voice and data connections versus data call arrival rate for $E_{b} / N_{0}=15,30$.

where $E\left[u_{i}\right]$ is the average number of class $i$ active connections,

$$
E\left[u_{i}\right]=\sum_{\mathbf{u} \in \mathcal{S}} u_{i} \pi(\mathbf{u}) .
$$

At the convergence of the fixed-point procedure, when $\lambda_{h, i}=$ $\lambda_{h, i}^{\text {(out) }}$, the steady-state behavior of the system is derived. 


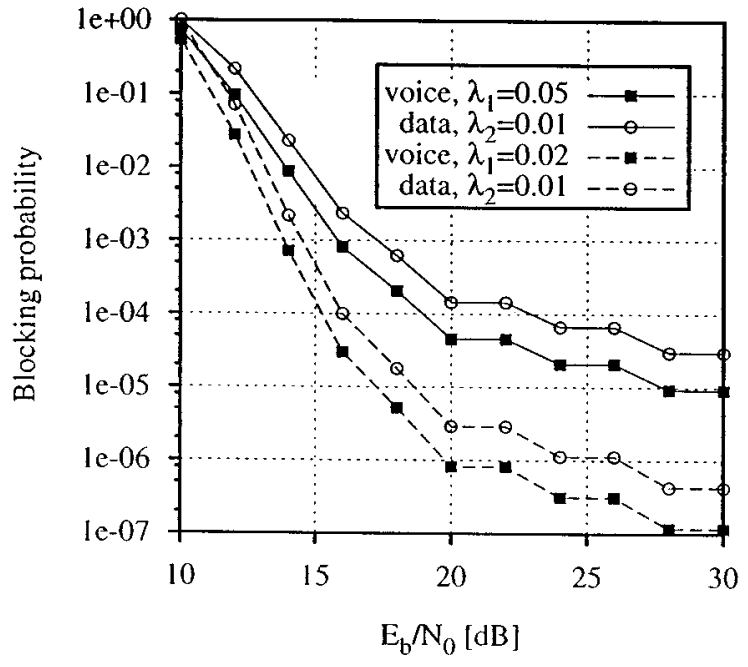

Fig. 4. Voice and data blocking probability versus $E_{b} / N_{0}$ for $\lambda_{2}=0.01$ and $\lambda_{1} / \lambda_{2}=2,5$.

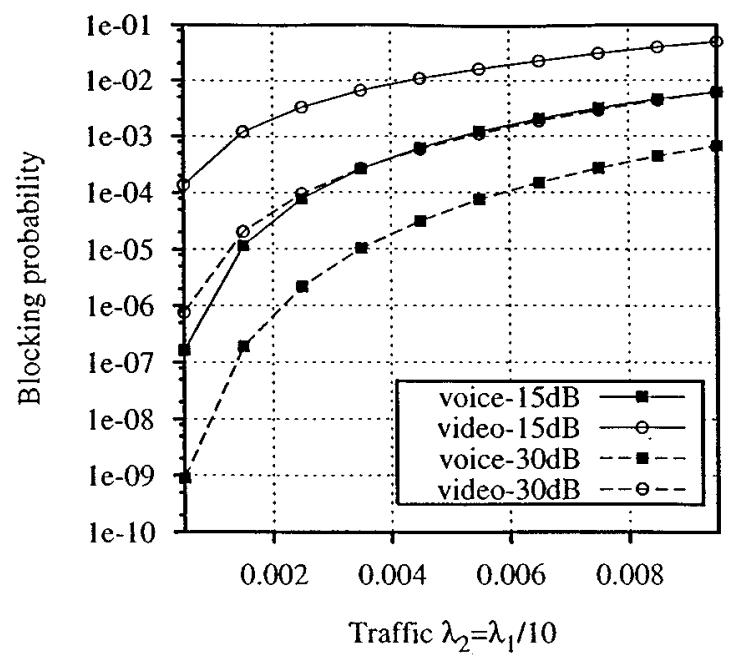

Fig. 5. Voice and video blocking probability versus video call arrival rate for $E_{b} / N_{0}=15,30$.

The call blocking probability for class $i$ calls, $P_{i}$, is computed as the sum of the probabilities of states in which the admission of a further class $i$ call would exceed the system capacity region defined by (2), i.e.,

$$
\begin{aligned}
& P_{i}=\sum_{\mathbf{u} \in \mathcal{S}_{i}} \pi(\mathbf{u}) \text { with } \\
& \mathcal{S}_{i}=\left\{\mathbf{u} \in \mathcal{S} \mid \sum_{\substack{j=1 \\
j \neq i}}^{C} \frac{u_{j}}{L_{j}}+\frac{u_{i}+1}{L_{i}}>K\right\} .
\end{aligned}
$$

In showing numerical results, we will consider $E\left[u_{i}\right]$ and $P_{i}$ as performance metrics.

\section{NUMERICAL RESULTS}

The first scenario we consider consists of a system which provides two classes of service: basic telephony and data connections aimed at transferring large data file. The spreading factor for voice service is the maximum one, $L_{1}=L_{\max }=256$; the average call duration is $1 / \mu_{1}=180 \mathrm{~s}$. Data service is provided at $30 \mathrm{~kb} / \mathrm{s}$ corresponding to spreading factor $L_{2}=128$. Assuming an average file size equal to $1 \mathrm{MB}$, the average duration of a data connection is $1 / \mu_{2}=267 \mathrm{~s}$. The limiting values of SIR are $\operatorname{SIR}_{1}^{*}=\operatorname{SIR}_{2}^{*}=10 \mathrm{~dB}$. We let the call arrival rates vary with a fixed ratio $\lambda_{2} / \lambda_{1}=1 / 5$. The average dwell time $1 / \mu_{h}$ is set to $90 \mathrm{~s}$.

Fig. 2 shows the voice and data call blocking probability versus traffic for different values of $E_{b} / N_{0}$. The blocking probability increases as the traffic intensity increases and as $E_{b} / N_{0}$ decreases. Due to the larger spreading factor, which causes smaller interference to other users, voice calls access the radio resources more easily than data connections.

Fig. 3 shows the average number of active voice and data calls for the same scenario.

Blocking probability for voice and data connections are compared in Fig. 4 versus $E_{b} / N_{0}$ when different ratios $\lambda_{2} / \lambda_{1}$ are considered. Data call arrival rate is equal to 0.01 requests per second, while voice call arrival rates is either equal to 0.05 or to 0.02 .

In the second scenario we consider a system which provides telephony and low bit-rate video conferencing. Telephony service has the same characteristics as in the previous scenario. Video conferencing bit rate is equal to $120 \mathrm{~kb} / \mathrm{s}$ using a spreading factor $L_{2}=32$. The average call duration is $1 / \mu_{2}=600 \mathrm{~s}$. As before, the limiting SIR is $10 \mathrm{~dB}$. The call arrival rate of voice requests is 10 times the arrival rate of video conference requests.

In Fig. 5 the voice and video blocking probability are plotted versus the video call arrival rate for different values of $E_{b} / N_{0}$.

\section{REFERENCES}

[1] D. Hong and S. Rappaport, "Traffic model and performance analysis for cellular mobile radio telephone systems with prioritized and nonprioritized handoff procedures," IEEE Trans. Veh. Technol., vol. VT-35, pp. 77-92, Aug. 1986.

[2] P. Tran-Gia and M. Mandjes, "Modeling of customer retrial phenomenon in cellular mobile networks," IEEE J. Select. Areas Commun., vol. SAC-15, pp. 1406-1414, Oct. 1997.

[3] K. K. Leung, W. A. Massey, and W. Whitt, "Traffic models for wireless communication networks," IEEE J. Select. Areas Commun., vol. SAC-12, pp. 1353-1364, Oct. 1994.

[4] Y. Lin, "Modeling techniques for large-scale PCS networks," IEEE Commun. Mag., vol. 35, no. 2, pp. 102-107, Feb. 1997.

[5] M. Meo and M. A. Marsan, "Approximate analytical models for dual-band GSM networks design and planning," in IEEE INFOCOM 2000, Tel-Aviv, Israel, Mar. 2000.

[6] A. M. Viterbi and A. J. Viterbi, "Erlang capacity of a power controlled CDMA system," IEEE J. Select. Areas Commun., vol. SAC-11, pp. 892-900, Aug. 1993.

[7] Y. Ishikawa and N. Umeda, "Capacity design and performance of call admission control in cellular CDMA systems," IEEE J. Select. Areas Commun., vol. SAC-15, pp. 1627-1635, Oct. 1997. 